\title{
A revised accumulated cyclone energy index
}

\author{
Jia-Yuh Yu, ${ }^{1}$ Chia Chou, ${ }^{2}$ and Ping-Gin $\mathrm{Chiu}^{3}$ \\ Received 19 May 2009; accepted 30 June 2009; published 30 July 2009.
}

[1] A new estimate of the wind energy associated with a tropical cyclone (TC), along with the revised accumulated cyclone energy (RACE) index, is introduced in this paper. In contrast to the conventional means employed in computing the ACE index, in which the wind energy is measured at the radius of maximum wind, the new estimate considers the mean wind energy averaged over a circular area based on the modified Rankine vortex structure. An examination of the seasonal TC activity using the JTWC best-track records over the western North Pacific suggests that, as long as there is a strong variability in TC activity (characterized by a substantial year-to-year variability in the RACE/ACE time series), employing a more precise estimate of the cyclone wind energy is not just physically reasonable, it can also be practically useful. Citation: Yu, J.-Y., C. Chou, and P.-G. Chiu (2009), A revised accumulated cyclone energy index, Geophys. Res. Lett., 36, L14710, doi:10.1029/ 2009GL039254.

\section{Introduction}

[2] Tropical cyclones, occurring over warm tropical oceans, are well known as the most powerful of all extreme weather systems on earth. Each year, approximately 88 tropical cyclones reaching tropical storm intensity occur around the globe [Henderson-Sellers et al., 1998]. The tropical cyclone (TC, hereafter) hazards come mainly from high-speed winds and heavy rainfalls that often cause massive losses of lives and properties to the invaded countries. Therefore, a skillful extended range forecast of seasonal TC activity in a particular basin is highly anticipated to mitigate these losses.

[3] Although predictions of seasonal TC activity in the Atlantic basin have demonstrated forecast skill since the mid-1980s [Gray, 1984], a more skillful prediction of TC activity several months prior to the beginning of the season remains a challenge for meteorologists, primarily because of great uncertainties associated with climate variability in the global atmosphere and ocean system, such as ENSO [Chen et al., 1998; Chan, 2000; Wang and Chan, 2002], decadal oscillation [Goldenberg et al., 2001; Yumoto and Matsuura, 2001], and global warming [Emanuel, 2005; Holland and Webster, 2007].

[4] The seasonal TC activity consists of frequency, path, lifetime, and strength of all TC systems occurring in the basin of interest. To understand how climate variability

\footnotetext{
${ }^{1}$ Department of Atmospheric Sciences, Chinese Culture University, Taipei, Taiwan.

${ }^{2}$ Research Center for Environmental Change, Academia Sinica, Taipei, Taiwan.

${ }^{3}$ Graduate Institute of Earth Science, Chinese Culture University, Taipei, Taiwan.

Copyright 2009 by the American Geophysical Union. 0094-8276/09/2009GL039254\$05.00
}

influences seasonal TC activity, a parameter (or index) suitable to describe the seasonal TC activity is demanded. Over the past few decades, several parameters have been proposed. The earliest and probably the most popular one is the frequency of TC which, in most cases, simply uses the annual number of TC geneses as a measure of the seasonal TC activity [e.g., Chen et al., 1998; Goldenberg et al., 2001]. The total TC lifetime, which considers the duration (in units of days) of all TC systems, is sometimes employed in combination with the annual TC number to characterize the seasonal TC activity [e.g., Wang and Chan, 2002; Emanuel, 2005].

[5] Recently, the "ACE" (accumulated cyclone energy) index was proposed by NOAA as an alternative measure of the total TC activity [Waple et al., 2001]. The ACE index inherently includes the duration and strength of TC by summing up the 6-hourly wind energy of all TC systems while they are at least the tropical storm strength. In ACE, the wind energy associated with a TC is assumed to be equivalent to the square of the maximum wind:

$$
K_{s m w}=v_{\max }^{2}
$$

where $v_{\max }$ is the maximum sustained wind speed.

[6] Since the radial structure of wind in a TC varies substantially depending on the strength [Weatherford and Gray, 1988a, 1988b; Mallen et al., 2005], the simple estimate in (1) may potentially lead to a spurious estimation of the TC activity. In this study, a new estimate of the wind energy based on the modified Rankine vortex, along with a revised ACE index, is introduced. Section 2 describes the data and mathematical algorithm used to derive the mean cyclone wind energy. Results are presented and discussed in section 3. Section 4 summarizes major findings.

\section{Data and Methodology}

\subsection{Data Source}

[7] The best tracks of TC archived in the Joint Typhoon Warning Center (JTWC) are employed to examine TC activity over the western North Pacific (WNP). This besttrack file contains locations and intensities (typically the maximum 1-minuite mean sustained wind) of each TC at 6hour intervals since 1949. It is noted that the radii of 35,50 , 65 , or 100 knots wind and the radius of maximum sustained wind became available in the best-track file since 2002 as additional information concerning TC structures. Since satellite monitoring of weather events was not available prior to 1965 , only the best-track records during the period 1965 2007 are analyzed.

\subsection{Algorithm for Cyclone Wind Energy}

[8] To include the radial wind structure in the estimate of cyclone wind energy, rather than its typical weighting at the 


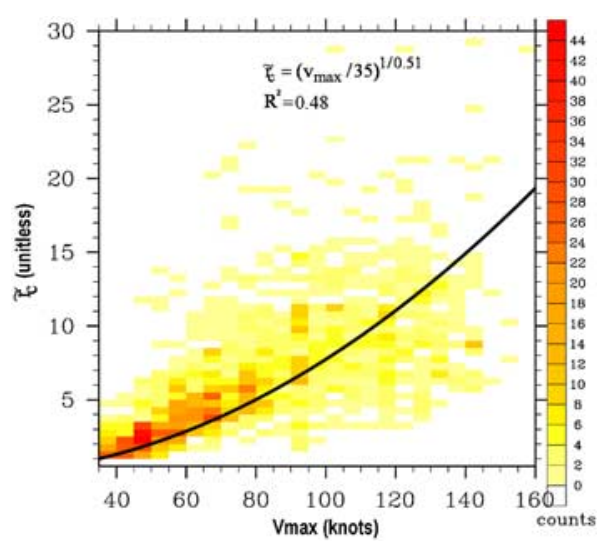

Figure 1. A mosaic plot of $\tilde{r}_{c}$ (non-dimensional cut-off radius) against $v_{\max }$ (maximum sustained wind) from 2767 JTWC 6-hourly best-track records over the WNP during 2002 2007. Color shadings represent the number of counts, while the solid line denotes the least squares regression curve using equation (9).

radius of maximum wind as in (1), the modified Rankine vortex structure is assumed:

$$
v(r)=\left\{\begin{array}{lll}
v_{\max }\left(r / r_{\max }\right) & , \quad r \leq r_{\max } \\
v_{\max }\left(r / r_{\max }\right)^{-\alpha}, & r>r_{\text {max }}
\end{array}\right.
$$

where $r$ denotes the distance from the center of vortex, $v(r)$ represents the tangential wind, $r_{\max }$ is the radius of maximum tangential wind, and $\alpha$ depicts the decaying tendency of wind outside $r_{\max }$.

[9] Following a similar approach as in (1), the wind energy of (2) gives

$$
K(r)= \begin{cases}v_{\max }^{2}\left(r / r_{\max }\right)^{2} & , \quad r \leq r_{\max } \\ v_{\max }^{2}\left(r / r_{\max }\right)^{-2 \alpha} & , \quad r>r_{\max }\end{cases}
$$

To facilitate latter analysis, we non-dimensionalize (3) to yield

$$
\tilde{K}(\tilde{r})= \begin{cases}\tilde{r}^{2} & , \quad \tilde{r} \leq 1 \\ \tilde{r}^{-2 \alpha} & , \quad \tilde{r}>1\end{cases}
$$

where the tilde variables (unitless quantities) are defined as below:

$$
\tilde{r}=r / r_{\max }, \quad \tilde{K}=K / K_{\max }, \quad K_{\max }=v_{\max }^{2}
$$

Integrating (4) over a circular area within a certain radius and dividing it by the same area, we obtain the mean wind energy

$$
\tilde{K}_{m r v}=\frac{1}{\pi \tilde{r}_{c}^{2}} \int_{0}^{\tilde{r}_{c}} \int_{0}^{2 \pi} \tilde{K}(\tilde{r}) \cdot \tilde{r} d \theta d \tilde{r}=\frac{1}{\tilde{r}_{c}^{2}}\left[\frac{1}{2}+\frac{\tilde{r}_{c}^{(2-2 \alpha)}-1}{(1-\alpha)}\right]
$$

where $\tilde{r}_{c}$ denotes the cut-off radius (unitless) within which the wind energy is estimated. The first and second terms in the bracket of (6) represent, respectively, the contributions from circulations within and outside $r_{\max }$.
[10] The dimensional form of (6) gives

$$
K_{m r v}=\frac{v_{\max }^{2}}{\tilde{r}_{c}^{2}}\left[\frac{1}{2}+\frac{\tilde{r}_{c}^{(2-2 \alpha)}-1}{(1-\alpha)}\right]
$$

Comparing (7) with (1), we note that $\tilde{K}_{m r v}$ represents a nonconstant rescaling factor of $K_{s m w}$ as the mean wind energy is considered. Since the JTWC maximum sustained wind speed within a TC is determined by averaging the sampled wind observations over a period of time, when it is applied in (7), the tangential and radial wind contributions to kinetic energy are both included.

\subsection{Relation Between $\tilde{\boldsymbol{r}}_{c}$ and $\boldsymbol{v}_{\max }$}

[11] To derive a relation between $\tilde{r}_{c}$ and $v_{\max }$, the lower equation of (2) is used:

$$
v(\tilde{r})=v_{\max } \tilde{r}^{-\alpha}
$$

Selecting a cut-off wind speed $\left(v_{c}\right),(8)$ yields

$$
\tilde{r}_{c}=\left(v_{\max } / v_{c}\right)^{1 / \alpha}
$$

where $\tilde{r}_{c}\left(=r_{c} / r_{\max }\right)$, the cut-off radius, is simply a multiple of $r_{\max }$. Here, we let $v_{c}=35$ knots in (9) such that only the circular area with the wind speed reaching at least the tropical storm magnitude is considered. Except for $\alpha=1$, we note that (9) depicts a nonlinear relation between $\tilde{r}_{c}$ and $v_{\max }$.

[12] Figure 1 shows the mosaic plot of $\tilde{r}_{c}$ against $v_{\max }$ using 2767 JTWC best-track records over the WNP during 2002 2007. The best-fit regression curve $\left(R^{2}=0.48\right)$ suggests that the best value for $\alpha$ is around 0.51 and $\tilde{r}_{c}$ increases with nearly the square of $v_{\max }$. We note that, with the aid of (9), this curve directly links $\tilde{r}_{c}$ to $v_{\max }$ such that (7) can be solved numerically. A new index resembling the conventional ACE index can be defined using (7) which is termed as the "revised ACE" (RACE) index in this study.

\section{Result and Discussion}

\subsection{Cyclone Wind Energy: $\boldsymbol{K}_{\text {sm } \boldsymbol{w}}$ Versus $\boldsymbol{K}_{\boldsymbol{m} r v}$}

[13] To contrast the differences between $K_{s m w}$ and $K_{m r v}$, Figure 2 first displays their unitless counterparts (i.e., $\tilde{K}_{s m w}$ and $\tilde{K}_{m r v}$ ) as a function of $v_{\max }$. This near-analytic solution shows that, except for small $v_{\max }, \tilde{K}_{s m w}$ is generally much larger than $\tilde{K}_{m r v}$ while their differences also tend to enlarge as $v_{\max }$ increases. It implies that, as compared to $\tilde{K}_{m r v}$ a spurious overestimation of the wind energy occurs for $\tilde{K}_{s m w}$ when it is applied, in particular, to measure a strong TC system.

[14] Figure 3 shows the accumulated wind energy (in units of $10^{4} \mathrm{knot}^{2} /$ year) patterns evaluated at each $5^{\circ} \times 5^{\circ}$ grid box over the WNP using the 6-hourly TC records during 1965 2007. It should be noted that summing up all values in Figure 3a (Figure 3b) is equal to the ACE (RACE) index. The energy pattern associated with $K_{s m w}$ (see Figure 3a) shows that intense TC activities $(>10)$ are narrowly confined over the subtropical WNP areas $\left(120 \sim 140^{\circ} \mathrm{E} / 15 \sim 25^{\circ} \mathrm{N}\right)$ with a maximum magnitude of 15.1 at $132.5^{\circ} \mathrm{E} / 17.5^{\circ} \mathrm{N}$. Despite large size differences, the 


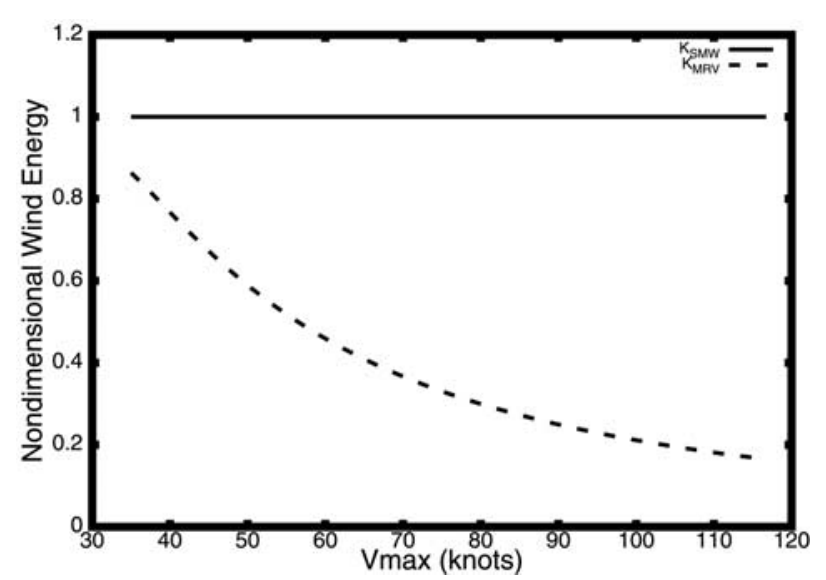

Figure 2. Magnitudes of non-dimensional cyclone wind energy as a function of $v_{\max }$ for $\tilde{K}_{s m w}$ (solid line) and $\tilde{K}_{m r v}$ (dotted line), respectively.

energy pattern associated with $K_{m r v}$ (see Figure 3b), nevertheless, exhibits a somewhat different picture featured by a little more widespread pattern. Large differences are
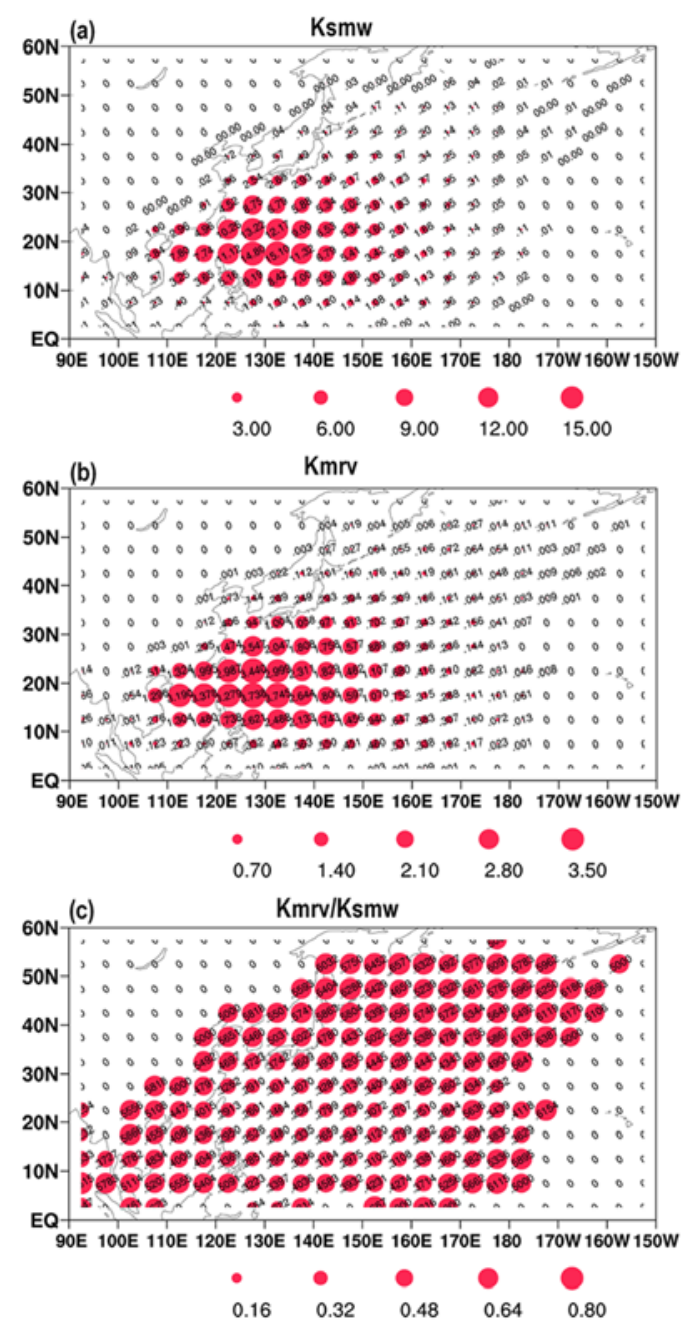

Figure 3. The accumulated wind energy (in units of $10^{4}$ $\mathrm{knot}^{2} /$ year) patterns measured at each $5^{\circ} \times 5^{\circ}$ grid box over the WNP associated with (a) $K_{s m w}$, (b) $K_{m r v}$, and (c) $K_{m r v}$ $K_{\text {smw }}$, respectively. noted especially over the South China Sea where stronger TC activity emerges when associated with $K_{m r v}$. The maximum accumulated wind energy associated with $K_{m r v}$ is just 3.745 (in units of $10^{4} \mathrm{knot}^{2} /$ year), about $1 / 4$ the size of the maximum $K_{s m w}$, consistent with the conjecture obtained in the near-analytic solution (Figure 2).

[15] The physical implication of the above results can be better explained by showing their ratios $\left(K_{m r v} / K_{s m w}\right)$ as in Figure 3c. As anticipated, smaller ratios $(<0.3)$ are confined over the subtropical WNP while larger ratios $(>0.5)$ dominate the basin perimeters, including the South China Sea. Since $K_{m r v} / K_{s m w}$ is identical to $\tilde{K}_{m r v}$, which tends to decrease as $v_{\max }$ increases, the areas with smaller (larger) ratios simply represent the dominance of wind energy by strong (weak) TC systems. Thus, the more narrowly confined $K_{s m w}$ pattern shown in Figure 3a is attributed to a spurious overestimation of TC activity in the subtropical WNP areas.

\subsection{Annual TC Activity: ACE Versus RACE}

[16] To access the impact of different estimate methods on seasonal TC activity, both the ACE and RACE time series are displayed in Figure 4a. Except for large size differences, the two time series are similar and highly correlated $\left(R^{2}=0.89\right)$. The mean ACE is about 300 (in units of $10^{4} \mathrm{knot}^{2} /$ year), compared to nearly 98 for the mean RACE. In addition, both ACE and RACE indices exhibit a strong year-to-year variability. During strong activity years (e.g., 1992, 1997, and 2004), the magnitudes of ACE (or RACE) can be several times larger than those during weak activity years (e.g., 1973, 1998 and 1999), a clear indication of climate control on TC activity.

[17] Similar to Figure 3c, Figure $4 b$ shows the RACE/ ACE time series to highlight their differences. Physically, the RACE/ACE ratio represents the yearly average of $K_{m r v} /$
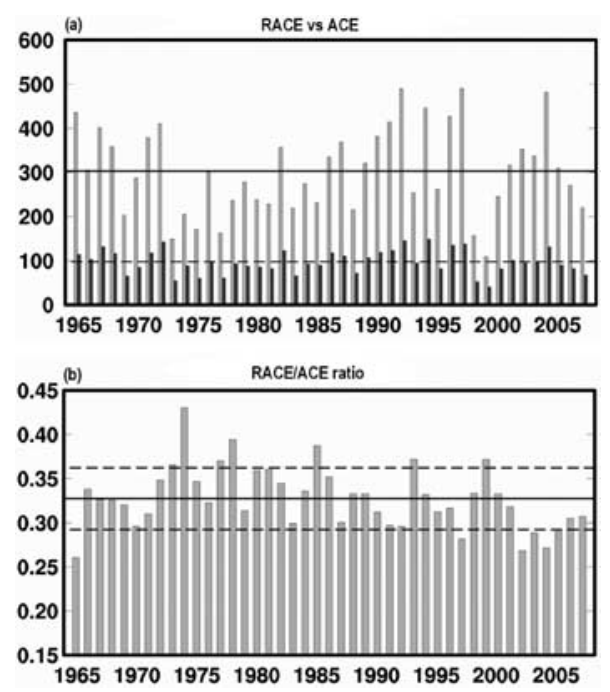

Figure 4. (a) Time series of the ACE (light histograms) and RACE indices (dark histograms) in the WNP basin (in units of $10^{4} \mathrm{knot}^{2} /$ year) with the solid (dashed) line denoting the mean ACE (RACE) index. (b) Time series of the RACE/ACE ratio with the solid line denoting the mean value. The upper (lower) dashed line represents one standard deviation above (below) the mean. 
Table 1. Total Lifetime of All TC Systems and Their Occurrence Frequencies Associated With the Tropical Storm, the Hurricane, and the Severe Hurricane Groups During the High-RACE/ACERatio Years ${ }^{\mathrm{a}}$

\begin{tabular}{lcccc}
\hline & $\begin{array}{c}\text { Total TC } \\
\text { Lifetime (Days) }\end{array}$ & $\begin{array}{c}\text { Tropical Storm } \\
(34 \sim 63 \mathrm{kt})\end{array}$ & $\begin{array}{c}\text { Hurricane } \\
(64 \sim 95 \mathrm{kt})\end{array}$ & $\begin{array}{c}\text { Severe Hurricane } \\
(\geq 96 \mathrm{kt})\end{array}$ \\
\hline 1973 & 85.3 & $60.4 \%$ & $30.2 \%$ & $9.4 \%$ \\
1974 & 140.5 & $65.3 \%$ & $31.7 \%$ & $3.0 \%$ \\
1977 & 95.8 & $65.5 \%$ & $22.7 \%$ & $11.8 \%$ \\
1978 & 147.8 & $67.7 \%$ & $26.0 \%$ & $6.3 \%$ \\
1985 & 135.3 & $56.6 \%$ & $39.4 \%$ & $4.0 \%$ \\
1993 & 150.8 & $65.0 \%$ & $24.5 \%$ & $10.5 \%$ \\
1999 & 65.5 & $59.2 \%$ & $31.3 \%$ & $9.5 \%$ \\
Average & 117.3 & $62.8 \%$ & $29.4 \%$ & $7.8 \%$ \\
\hline
\end{tabular}

${ }^{\mathrm{a}}$ The high-ratio years denote the years with the RACE/ACE ratio at least one standard deviation above the mean.

$K_{s m w}$ (i.e., $\tilde{K}_{m r v}$ ) and a low- (high-) ratio year implies the dominance of strong (weak) TC systems in that year. We note that if the yearly RACE/ACE ratios are somehow relatively steady, the TC activity of various strengths will be evenly distributed in every year. Under this circumstance, employing the RACE index to measure the seasonal TC activity will become practically meaningless since it is just a constant rescaling factor of the conventional ACE index. Surprisingly, a notable year-to-year variability exists in the RACE/ACE time series which implies a substantial variation in the TC activity of various strengths during the studied period.

[18] To elaborate the cause responsible for such variability, the years with significantly high and low RACE/ACE ratios are compared. Here, a high-ratio (low-ratio) year refers to one in which the RACE/ACE ratio is at least one standard deviation above (below) the mean. Tables 1 and 2 summarize the TC activity during high-ratio and low-ratio years, respectively. For convenience, the TC lifetime is divided into three groups, namely the "tropical storm" (35 64 knots), the "hurricane" (65 94knots), and the "severe hurricane" ( $\geq 95$ knots) groups, according to the intensity. In general, the total TC lifetime in high-ratio years is much shorter than that in low-ratio years (averages 117 vs. 223 days/year). During high-ratio years (Table 1), the occurrence frequency associated with tropical storm group accounts for, on average, $62.8 \%$ of the total TC lifetime, followed by $29.4 \%$ associated with hurricane group, and $7.8 \%$ associated with severe hurricane group. During lowratio years (Table 2) the occurrence frequency associated with tropical storm group accounts for just $39.9 \%$ of the total TC lifetime, a marked $22.9 \%$ drop in frequency as compared to Table 1. Conversely, a significant increase of the occurrence frequency associated with severe hurricane group is noticed (from 7.8 to $27.5 \%$ ) while the change associated with hurricane group seems to be rather small (from 29.4 to $32.6 \%$ ). These results clearly demonstrate that the irregular dominance by strong (or weak) TC systems in yearly TC activity is responsible for the RACE/ACE variability shown in Figure 4b.

[19] While the physical implication of RACE/ACE may be clear enough, its link to climate variability still needs to be clarified. It was shown by previous studies that the ENSO effect on TC activity may be important over the WNP [Chan, 2000; Wang and Chan, 2002]. To find out whether the ENSO signal exists in RACE/ACE, we obtain the El Niño (EN) and La Niña (LN) years from the ENSO monitoring homepage of the Climate Prediction Center. During 1965 2007, 11 EN years $(1965,69,72,82,87,91$, $92,94,97,02$ and 04$)$ and $8 \mathrm{LN}$ years $(1971,73,74,75,85$, 88,99 and 00) are identified. When these years are compared with those in Tables 1 and 2, the link between RACE/ACE and ENSO emerges. For example, for the 7 high-ratio years listed in Table 1, 4 of them $(1973,74,85$, and 99) are associated with the LN years. Similarly, for the 6 low-ratio years listed in Table 2, 4 of them $(1965,97,02$, and 04 ) belong to the EN years. However, discrepancies are also noted in some years (e.g., 1977, 93 and 05) in which the relation between ENSO and RACE/ACE seems to be rather small. It clearly suggests that while the ENSO effect may be important, it is not the only factor that determines the RACE/ACE variability, consistent with the finding of Chan [2000].

\section{Summary}

[20] In this paper, a new estimate of the wind energy associated with a TC is introduced. In contrast to the conventional means used in computing the ACE index, in which the wind energy is measured at the radius of maximum wind $\left(K_{s m w}\right)$, the new estimate considers the mean wind energy averaged over a circular area based on the modified Rankine vortex $\left(K_{m r v}\right)$. To contrast the differences between $K_{s m w}$ and $K_{m r v}$ the JTWC best-track records over the WNP during 1965 2007 are analyzed. The near-analytic solution shows that while $K_{s m w}$ is always larger than $K_{m r r}$, their differences enlarge quickly as the intensity of TC increases. A comparison between them indicates that the conventional estimate (i.e., $K_{s m w}$ ) may have overestimated TC activities over the subtropical WNP areas where strong TC systems were frequently observed.

[21] A revised ACE (RACE) index based on $K_{m r v}$ is also introduced in this study as an alternative means for describing the total TC activity. Despite a striking similarity between the ACE and RACE time series $\left(R^{2}=0.89\right)$, differences between them (featured by a notable year-toyear variability in the RACE/ACE time series) exist. Examining TC activity of various strengths indicates that such variability is attributed to the irregular dominance by strong (or weak) TC systems in yearly TC activity. Thus we may conclude that, as long as there is a strong variability in TC activity, employing a more precise estimate of the cyclone wind energy is not just physically reasonable, it can also be practically useful.

Table 2. Same as in Table 1, but for the Low-Ratio Years ${ }^{\mathrm{a}}$

\begin{tabular}{lcccc}
\hline & $\begin{array}{c}\text { Total TC } \\
\text { Lifetime (Days) }\end{array}$ & $\begin{array}{c}\text { Tropical Storm } \\
(34 \sim 63 \mathrm{kt})\end{array}$ & $\begin{array}{c}\text { Hurricane } \\
(64 \sim 95 \mathrm{kt})\end{array}$ & $\begin{array}{c}\text { Severe Hurricane } \\
(\geq 96 \mathrm{kt})\end{array}$ \\
\hline 1965 & 164.0 & $47.3 \%$ & $26.5 \%$ & $26.2 \%$ \\
1997 & 200.8 & $50.2 \%$ & $29.5 \%$ & $20.3 \%$ \\
2002 & 157.0 & $40.8 \%$ & $34.0 \%$ & $25.2 \%$ \\
2003 & 139.3 & $45.0 \%$ & $32.9 \%$ & $22.1 \%$ \\
2004 & 406.5 & $26.8 \%$ & $36.7 \%$ & $36.5 \%$ \\
2005 & 270.8 & $29.7 \%$ & $35.7 \%$ & $34.6 \%$ \\
Average & 222.9 & $39.9 \%$ & $32.6 \%$ & $27.5 \%$ \\
\hline
\end{tabular}

${ }^{\mathrm{a}}$ The RACE/ACE ratio is at least one standard deviation below the mean. 
[22] Acknowledgments. This work was sponsored by the National Science Council of Taiwan under grants NSC96-2745-M-034-001-URD and NSC97-2682-M-001-002. The authors thank the two anonymous reviewers for their valuable comments.

\section{References}

Chan, J. C. L. (2000), Tropical cyclone activity over the western North Pacific associated with El Niño and La Niña events, J. Clim., 13, 2960 2972, doi:10.1175/1520-0442(2000)013<2960:TCAOTW > 2.0.CO;2.

Chen, T.-C., S.-P. Weng, N. Yamazaki, and S. Kiehne (1998), Interannual variation in the tropical cyclone formation over the western North Pacific, Mon. Weather Rev., 126, 1080-1097, doi:10.1175/1520-0493(1998) $126<1080$ :IVITTC $>2.0 . \mathrm{CO} ; 2$

Emanuel, K. (2005), Increasing destructiveness of tropical cyclones over the past 30 years, Nature, 436, 686-688, doi:10.1038/nature03906.

Goldenberg, S. B., C. W. Landsea, A. M. Mestas-Nunez, and W. M. Gray (2001), The recent increase in Atlantic hurricane activity: Causes and implications, Science, 293, 474-479, doi:10.1126/science.1060040.

Gray, W. M. (1984), Atlantic seasonal hurricane frequency: Part II: Forecasting its variability, Mon. Weather Rev., 112, 1669-1683, doi:10.1175 1520-0493(1984)112<1669:ASHFPI > 2.0.CO;2.

Henderson-Sellers, A., et al. (1998), Tropical cyclones and global climate change: A post-IPCC assessment, Bull. Am. Meteorol. Soc., 79, 19-38, doi:10.1175/1520-0477(1998)079<0019:TCAGCC > 2.0.CO;2.

Holland, G. J., and P. J. Webster (2007), Heightened tropical cyclone activity in the North Atlantic: Natural variability or climate trend?, Philos. Trans. R. Soc., Ser. A, 365, 2695-2716, doi:10.1098/ rsta.2007.2083
Mallen, K. J., M. T. Montgomery, and B. Wang (2005), Reexamining the near-core radial structure of the tropical cyclone primary circulation: Implications for vortex resiliency, J. Atmos. Sci., 62, 408-425, doi:10.1175/JAS-3377.1

Wang, B., and J. C. L. Chan (2002), How strong ENSO events affect tropical storm activity over the western North Pacific, J. Clim., 15, 1643-1658, doi:10.1175/1520-0442(2002)015<1643:HSEEAT > 2.0.CO;2.

Waple, A. M., et al. (2001), Climate assessment for 2001, Bull. Am. Meteorol. Soc., 83, S1-S62.

Weatherford, C. L., and W. M. Gray (1988a), Typhoon structure as revealed by aircraft reconnaissance. Part I: Data analysis and climatology, Mon. Weather Rev., 116, 1032-1043, doi:10.1175/1520-0493(1988) $116<1032$ :TSARBA $>2.0 . \mathrm{CO} ; 2$.

Weatherford, C. L., and W. M. Gray (1988b), Typhoon structure as revealed by aircraft reconnaissance. Part II: Structure variability, Mon. Weather Rev., 116, 1044-1056, doi:10.1175/1520-0493(1988)116<1044:TSAR$\mathrm{BA}>2.0 . \mathrm{CO} ; 2$

Yumoto, M., and T. Matsuura (2001), Interdecadal variability of tropical cyclone activity in the western North Pacific, J. Meteorol. Soc. Jpn., 79, 23-35, doi:10.2151/jmsj.79.23.

P.-G. Chiu, Graduate Institute of Earth Science, Chinese Culture University, Taipei 11114, Taiwan.

C. Chou, Research Center for Environmental Change, Academia Sinica, Taipei 11529, Taiwan

J.-Y. Yu, Department of Atmospheric Sciences, Chinese Culture University, Taipei 11114, Taiwan. (jiayuh@atmos.pccu.edu.tw) 